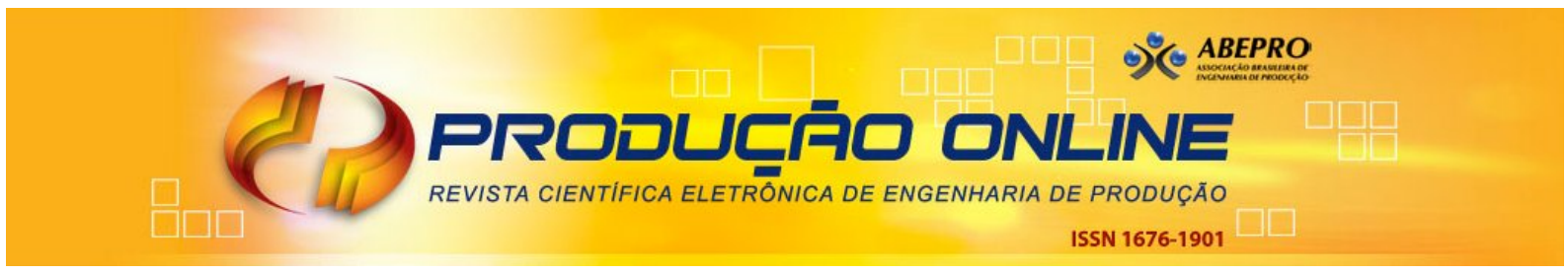

\title{
GERAÇÃO DE VALOR NO CONTEXTO DA ESTRATÉGIA DE MANUFATURA: UMA ANÁLISE EM MANUFATURAS CALÇADISTAS
}

\section{VALUE GENERATION IN THE CONTEXT OF MANUFACTURING STRATEGY: AN ANALYSIS IN SHOE MANUFACTORIES}

\author{
Sergio Evangelista Silva* E-mail: sergio@deenp.ufop.br \\ Flávio César Faria Fernandes** E-mail: dfcf@power.ufscar.br \\ Edemilson Nogueira** E-mail: edn@dep.ufscar.br \\ * Instituto de Ciências Exatas e Aplicadas (UFOP), João Monlevade - MG \\ **Universidade Federal de São Carlos (UFSCar), São Carlos, SP
}

\begin{abstract}
Resumo: A função manufatura tem como propósito oferecer produtos que gerem valor para os clientes. Todavia, esta não é uma tarefa exclusiva desta função, para esse fim ela deve atuar de forma conjunta com as funções marketing e vendas (M\&V) e pesquisa e desenvolvimento (P\&D). Este artigo denomina estas três funções de geradoras de valor e discute o seu papel mútuo no processo de geração de valor dos produtos de uma empresa. A validação deste modelo é realizada por meio de três estudos de caso comparativos, realizados em manufaturas calçadistas (MCs) com diferentes estratégias de geração de valor. O estudo empírico permitiu comprovar o papel mútuo das funções estudadas de geração de valor do produto, bem como verificar que a configuração e o papel relativo de cada função muda conforme a estratégia adotada pela empresa.
\end{abstract}

Palavras-chave: Estratégia de Manufatura. Valor do Produto. Estratégia Competitiva. Funções Geradoras de Valor. Indústria Calçadista.

\begin{abstract}
The manufacturing function has the role of provide to the customers products with generate value. However this task is not unique of this function, it has to work with the functions research and development (R\&D) and marketing and sales (M\&S). This paper call these three functions as generating value functions, and discuss their mutual role in the process of generating value in the products of a firm. The validation of this model is performed by three comparative case studies, conducted in three shoe manufactories (SMs), with different value generation strategies. The empirical study shows that the mutual role of generating value functions in the value generating process, and that the configuration and the relative role of each function change according to the firm strategy followed.
\end{abstract}

Keywords: Manufacturing strategy. Product value. Competitive strategy. Generating value functions. Footwear industry.

\section{INTRODUÇÃO}

A competitividade de uma empresa está intimamente associada à sua capacidade de gerar valor em seus produtos, (BROWMAN; AMBROSINI, 2007; PORTER, 1989). Segundo Santala e Parvinen (2007), o conceito de valor para o cliente é essencial para se entender a lógica das empresas, estando diretamente relacionado à estratégia. Um dos modelos mais conhecidos a este respeito é o da 
"Cadeia de Valor" proposto por Porter (1989). Este autor decompõe a firma em atividades de apoio (infra-estrutura; recursos humanos; tecnologia; e compras) e essenciais (logística interna; operações; logística externa; marketing e vendas; e serviço), em que estas últimas são diretamente responsáveis pelo processo qualificado como geração de valor. Há autores que consideram como primárias as funções manufatura, marketing \& vendas (M\&V) e finanças, uma vez que todas as empresas industriais possuem essas três funções; já outros consideram como primárias as funções manufatura, $M \& V$ e pesquisa e desenvolvimento (P\&D), uma vez que elas interagem diretamente com a estratégia da unidade de negócio. Para Wheelwright (1984), é possível identificar em empresas manufatureiras três níveis estratégicos: o corporativo, o do negócio e o funcional; a estratégia corporativa se decompõe em diversas estratégias de negócios, em que cada uma é decomposta nas estratégias funcionais de: manufatura, $M \& V, P \& D$ e financeira, dentre outras.

Fleury e Fleury (2003) consideram que a estratégia de uma firma está baseada em uma das três competências, a saber: a excelência operacional, a inovação em produto, ou a relação com o cliente, que têm sua origem respectivamente nas funções manufatura, $M \& V$ e $P \& D$. Em consonância com esses pressupostos, este artigo tem como objetivo analisar o papel mútuo das funções manufatura, M\&V e P\&D no processo de geração de valor de manufaturas calçadistas. Vale destacar que este processo perpassa as diferentes funções organizacionais, exigindo delas um funcionamento coordenado (VARANDAS JR.; MIGUEL, 2012; OLAVARRIETA; FRIEDMANN, 2008).

Como objeto de estudo e validação da teoria são apresentados três estudos de caso em manufaturas calçadistas (MCs), localizadas em Franca, interior do estado de São Paulo. O método de pesquisa é o estudo de casos múltiplos com abordagem qualitativa.

Além desta seção introdutória o artigo encontra-se organizado da seguinte maneira, na segunda seção é apresentado o referencial teórico, na terceira o método utilizado, nas seções quatro e cinco são apresentados respectivamente os dados da pesquisa e a análise, e na sexta seção a conclusão do estudo. 


\section{REFERENCIAL TEÓRICO}

Baseado em Pilkington e Meredith (2009) pode-se concluir que a literatura de estratégia de manufatura tem seu marco fundamental em Skinner (1969); no final da década de 1960 este autor aponta que a crise de competitividade da indústria norteamericana vivida naquele momento era devida a uma ênfase da função manufatura somente na redução de custos. Este autor defendia na ocasião de sua pesquisa a elaboração de uma "política de manufatura", em que além dos custos deveriam ser considerados aspectos como entrega rápida, qualidade, confiabilidade na entrega (pontualidade), flexibilidade em se ajustar à demanda, e em introduzir novos produtos rapidamente.

Posteriormente Skinner (1974) defendeu a construção de um foco estratégico, ou seja, a priorização de um conjunto restrito de quesitos pela manufatura, conceito este amplamente adotado desde então (BACHEGA; GODINHO FILHO, 2011; HALLGREN; OLHAGER, 2006; HILL, 1994). A definição deste foco deve ocorrer por causa da existência de trade-offs no sistema de produção no curto e médio prazos (PEREIRA; SELLITTO, BORCHARDT, 2010; MAIA et al. 2005), que todavia podem ser rompidos por meio do avanço tecnológico no longo prazo (PAIVA et al. 2004), ou pela mudança de paradigma produtivo (GODINHO FILHO; FERNANDES, 2005).

Com efeito, a função manufatura deve eleger uma quantidade restrita de objetivos de desempenho (SLACK, 2002), procurando obter neles performance superior à de seus concorrentes.

\subsection{Estratégia de manufatura e estratégia}

Baseado em Ronda-Pupo e Guerras-Martin (2012) pode-se afirmar que o desafio da teoria da estratégia é relacionar elementos do plano semântico e valorativo à realidade dos objetos sobre os quais ela se desenvolve. No contexto da manufatura a dualidade entre o semântico e o real revela-se respectivamente nos termos objetivos de desempenho e recursos disponíveis (PEIXOTO et al. 2013; SILVA et al. 2012).

A estratégia pode ser elaborada nos níveis: corporativo, da unidade estratégica de negócio (UEN) e funcional (SLACK, 2002; HAYES; WHEELWRIGHT, 
1984). A estratégia corporativa trata de aspectos como a política de diversificação do portfólio de ativos, e a obtenção de sinergias entre as empresas de um mesmo grupo - a corporação (ANSOFF, 1990).

A estratégia da UEN envolve a inserção da unidade produtiva na estratégia corporativa (JOSEPH; OCASIO, 2012; PEHRSSON, 2006; CHANDLER, 1990), em que são tratados aspectos como o seu comportamento em relação à influência dos agentes do ambiente (PORTER, 1986), a configuração dos seus recursos (FINNEY et al. 2008; BARNEY, 1991), e a estratégia competitiva, que define como a ela enfrentará seus concorrentes (PERTUSA-ORTEGA et al. 2009; PORTER, 1986).

A estratégia de manufatura encontra-se no nível funcional em que esta função deve definir como apoiar a estratégia da UEN na qual está inserida (ABE; CARVALHO, 2011; BROWMN; BLACKMON, 2005). Um aspecto relevante neste processo é definir como serão configurados os recursos desta função.

Hayes e Wheelwright (1984) classificam os recursos da manufatura em estruturais e infra-estruturais, que consistem respectivamente nos elementos tangíveis e nas regras gerenciais do sistema de produção. Peixoto et al. (2013) consideram que o alcance de um conjunto de objetivos de desempenho exige uma determinada configuração destes recursos. Todavia, como aponta estudo de Guimarães et al. (2014) a definição dos objetivos dependerá fortemente do mercado em que a empresa se encontra.

No Quadro 1 são apresentados diversos objetivos de desempenho apontados por vários autores. Nos casos em que o texto de referência é uma pesquisa empírica é apresentada a indústria objeto do estudo, a não apresentação de uma indústria associada ao trabalho indica que a referência possui abordagem teórica. Embora sejam vários os objetivos de desempenho pode-se observar que os mais citados são o custo, a entrega, a flexibilidade e a qualidade. Isso provavelmente ocorre porquê textos seminais sobre a estratégia de manufatura (ex. HILL,1994; ROTH; MILLER, 1994) convergiram para estes parâmetros, sendo referência para muitos dos textos posteriores. Deve-se ressaltar que a terminologia de objetivos de desempenho presente na literatura é bastante variada, todavia, a classificação proposta no Quadro 1 considerou a definição de cada autor à luz dos termos mais citados, sendo portanto utilizada a terminologia destes últimos como referência.

Além dos quatro objetivos destacados, ressalta-se a importância atribuída por vários textos ao objetivo serviço, que consiste em ações relativas ao atendimento do 
cliente, divulgação e estrutura de pós-venda. Outro objetivo que tem ganho relevância é o desempenho ambiental, que ocorre quando a empresa utiliza ações relativas a preservação do meio ambiente como forma de agregar valor ao produto, buscando a preferência de clientes que valorizam este aspecto (CONTADOR, 2003). Por fim, o enfoque de alguns textos em apenas um objetivo indica que este é o tema central abordado, não implicando na desconsideração dos demais.

Quadro 1 - Síntese dos objetivos de desempenho segundo diversos autores

\begin{tabular}{|c|c|c|c|c|c|c|c|c|c|c|c|c|c|c|c|c|}
\hline & 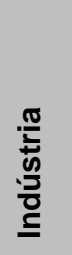 & $\begin{array}{l}\frac{0}{0} \\
\frac{\pi}{0} \\
\frac{\overline{0}}{0} \\
\frac{\pi}{\pi} \\
\frac{0}{0} \\
\frac{\pi}{0}\end{array}$ & $\begin{array}{l}\frac{0}{0} \\
\frac{\pi}{0} \\
\frac{0}{0} \\
\frac{\pi}{0} \\
\frac{0}{\pi} \\
0\end{array}$ & 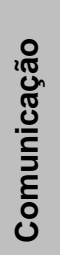 & $\frac{0}{0}$ & $\begin{array}{l}\overline{0} \\
\frac{\pi}{0} \\
: \frac{}{0} \\
\overline{0} \\
\frac{\pi}{0} \\
\frac{1}{0} \\
0 \\
0 \\
0 \\
0\end{array}$ & 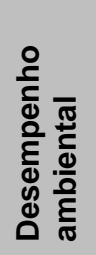 & 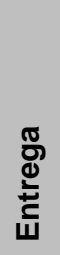 & 离 & $\begin{array}{l}\frac{0}{0} \\
\frac{\pi}{0} \\
\frac{0}{\overline{0}} \\
\bar{x} \\
\frac{0}{4}\end{array}$ & 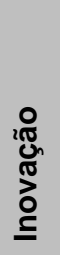 & $\begin{array}{l}\frac{0}{0} \\
\frac{\pi}{0} \\
\frac{\pi}{\pi} \\
\frac{\pi}{2}\end{array}$ & 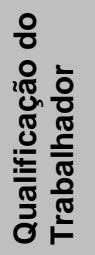 & 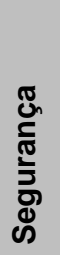 & $\frac{0}{2}$ & $\begin{array}{l}\frac{\pi}{\delta} \\
\frac{0}{0} \\
\frac{c}{0} \\
\stackrel{-}{-}\end{array}$ \\
\hline SKINNER, 1969 & - & & & & $x$ & & & $\mathrm{x}$ & & $\mathrm{x}$ & & $\mathrm{x}$ & & & & \\
\hline SKINNER, 1974 & - & & & & $\mathrm{x}$ & & & $\mathrm{x}$ & & $\mathrm{x}$ & & $\mathrm{x}$ & & & & \\
\hline $\begin{array}{l}\text { HAYESWHEELWR } \\
\text { IGHT, } 1984\end{array}$ & $\mathrm{D}$ & & & & $x$ & & & $x$ & & $x$ & & $x$ & & & & \\
\hline HILL, 1994 & & & & & $x$ & & & $\mathrm{x}$ & & $\mathrm{x}$ & & $x$ & & & & \\
\hline $\begin{array}{l}\text { ROTH; MILLER, } \\
1994\end{array}$ & $\mathrm{D}$ & & & & $x$ & & & $x$ & & $x$ & & $x$ & & & $x$ & \\
\hline $\begin{array}{l}\text { MacCARTHY; } \\
\text { FERNANDES, } \\
2000\end{array}$ & - & $x$ & & & & & & $x$ & & $x$ & & & & & & \\
\hline CONTADOR, 2003 & - & & & $\mathrm{x}$ & $\mathrm{x}$ & & $x$ & $x$ & $\mathrm{x}$ & $\mathrm{x}$ & $\mathrm{x}$ & & & & $x$ & \\
\hline $\begin{array}{l}\text { KLIPPEL; VALLE; } \\
\text { LAUREANO, } 2005\end{array}$ & ME & & & & $x$ & & & $x$ & & $\mathrm{x}$ & & $x$ & & & $x$ & \\
\hline $\begin{array}{l}\text { SELLITTO; } \\
\text { WALTER, } 2005\end{array}$ & $\mathrm{M}$ & & $x$ & & & & & $x$ & $x$ & & & & $x$ & & & \\
\hline $\begin{array}{l}\text { SILVA; SANTOS, } \\
2005\end{array}$ & $\mathrm{MO}$ & & & & $x$ & & & $x$ & & $x$ & & $x$ & & & & \\
\hline $\begin{array}{l}\text { SELLITTO; } \\
\text { WALTER, } 2006\end{array}$ & $E$ & & & $x$ & $\mathrm{x}$ & & & $x$ & & & & $x$ & & & $x$ & $x$ \\
\hline $\begin{array}{l}\text { SILVA; SANTOS, } \\
2008\end{array}$ & - & & & & $\mathrm{x}$ & & $x$ & $x$ & & $x$ & & $x$ & & & $x$ & $x$ \\
\hline $\begin{array}{l}\text { BORCHARDT et al. } \\
2010\end{array}$ & $\mathrm{C}$ & & & & & & $x$ & & & & & & & & & \\
\hline $\begin{array}{l}\text { CALIFE; } \\
\text { NOGUEIRA; } \\
\text { ALVES FILHO, } \\
2010\end{array}$ & L B & & & & $x$ & & & $x$ & & $x$ & & $x$ & & & $x$ & \\
\hline $\begin{array}{l}\text { PEREIRA; } \\
\text { SELLITO; } \\
\text { BORCHARDT, } \\
2010 \\
\end{array}$ & $\mathrm{C}$ & & & & $x$ & & & $x$ & & $x$ & & $x$ & & & $x$ & \\
\hline $\begin{array}{l}\text { SANTOS; } \\
\text { BATALHA, } 2010\end{array}$ & $\mathrm{CE}$ & & & & $x$ & & & $x$ & & $x$ & & $x$ & & & & \\
\hline $\begin{array}{l}\text { SILVA; SANTOS; } \\
\text { CASTRO, } 2010\end{array}$ & $\mathrm{MO}$ & & & & $x$ & & & $x$ & & $x$ & & $x$ & & & & \\
\hline
\end{tabular}

Fonte: Os autores 


\begin{tabular}{|c|c|c|c|c|c|c|c|c|c|c|c|c|c|c|c|c|}
\hline & 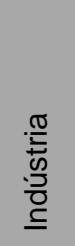 & $\begin{array}{l}\frac{0}{0} \\
\frac{\pi}{0} \\
\frac{0}{\overline{0}} \\
\frac{\pi}{0} \\
\frac{0}{\pi} \\
\frac{\pi}{0}\end{array}$ & $\begin{array}{l}\frac{0}{0} \\
\frac{\pi}{0} \\
\frac{0}{0} \\
\frac{0}{\pi} \\
0\end{array}$ & 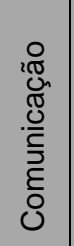 & $\frac{O}{n}$ & 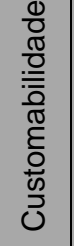 & 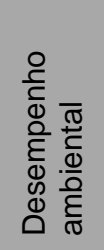 & 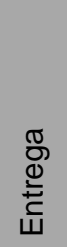 & 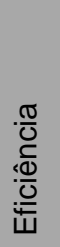 & $\begin{array}{l}\frac{0}{0} \\
\frac{\pi}{0} \\
\frac{0}{\overline{0}} \\
\frac{\pi}{\pi} \\
\frac{0}{4}\end{array}$ & 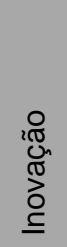 & $\begin{array}{l}\frac{0}{\pi} \\
\frac{\pi}{0} \\
\frac{0}{\bar{\sigma}} \\
0 \\
0\end{array}$ & 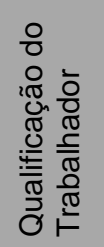 & 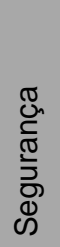 & 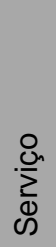 & $\begin{array}{l}\frac{\pi}{8} \\
\frac{0}{0} \\
\frac{0}{0} \\
0 \\
\end{array}$ \\
\hline $\begin{array}{l}\text { ALVES } \\
\text { FILHO; } \\
\text { NOGUEIRA; } \\
\text { BENTO, } 2011\end{array}$ & MT & & & & $\mathrm{x}$ & & & $x$ & & $x$ & & $x$ & & & & \\
\hline $\begin{array}{l}\text { BACHEGA; } \\
\text { GODINHO } \\
\text { FILHO, } 2011\end{array}$ & C & $x$ & & & & $x$ & & $x$ & & $x$ & & $x$ & & & & \\
\hline $\begin{array}{l}\text { SELLITTO et } \\
\text { al. } 2011\end{array}$ & $E$ & & & & & & & $x$ & & & & & & & $x$ & \\
\hline $\begin{array}{l}\text { SILVA; } \\
\text { SANTOS; } \\
\text { CASTRO; } \\
2012 \\
\end{array}$ & $\mathrm{MO}$ & & & & $x$ & & & $x$ & & $x$ & & $x$ & & & & \\
\hline $\begin{array}{l}\text { SILVA et al. } \\
2012\end{array}$ & \begin{tabular}{|l|}
$P Q$ \\
\end{tabular} & & & $x$ & $x$ & & & $x$ & & $x$ & $x$ & $x$ & & $x$ & & \\
\hline $\begin{array}{l}\text { BORCHARDT } \\
\text { et al. } 2012\end{array}$ & $\mathrm{Q}$ & & & & & & $x$ & & & & & & & & & \\
\hline $\begin{array}{l}\text { PEIXOTO et } \\
\text { al. } 2013\end{array}$ & $\begin{array}{ll}C E \\
\end{array}$ & & & & $\mathrm{x}$ & & & $x$ & & $x$ & & $x$ & & & $x$ & \\
\hline $\begin{array}{l}\text { GUIMARAES } \\
\text { et al. } 2014\end{array}$ & $A$ & & & & $x$ & & & $x$ & & $x$ & & $x$ & & & & \\
\hline
\end{tabular}

Fonte: Os autores.

\subsection{0 papel da estratégia de manufatura na geração de valor}

A competitividade da empresa industrial baseia-se na sua capacidade de gerar valor para o cliente por meio dos produtos que fabrica (ADNER et al. 2006). A ideia de valor transcende os aspectos pecuniários e concretos relativos ao bem, devendo ser compreendida a partir do trinômio objeto, ambiente-mediador e cliente. O objeto constitui-se no produto, que possui funcionalidades que justificam a sua criação, bem como as características que traduzem sua performance (projeto, tempo de resposta, qualidade...) (CACHON; SWINNEY, 2011; KIM; CHHAJED, 2002; MEREDITH et al. 1994). O ambiente-mediador da compra consiste nos elementos referentes à circunstância da mesma, tais como, o conteúdo informacional do produto, sua apresentação, o atendimento, os aspectos circunstanciais, dentre outros (KALRA et al. 2003; BABIN et al. 1994). Por fim, o cliente constitui-se no agente decisor do processo de compra. 
O valor do produto possui natureza dicotômica (CRONIN et al. 1997) em que de um lado tem-se a realidade concreta (o objeto), e de outro, a subjetividade do cliente, ou seja, suas necessidades, percepções, condição e preferências. Com efeito, o valor de um produto é conferido pela performance deste em uma série de atributos, relativos a estes dois aspectos, sendo denominados atributos de valor.

A presença da subjetividade na atribuição de valor a um produto faz com que este seja avaliado não apenas nos seus termos concretos, mas também segundo aspectos psicossociais, como reputação, risco iminente, imagem de marca, estilo de vida, identificação com grupos sociais, atendimento, dentre outros (AUGER et al.2010;).

Nota-se que o conceito de objetivo de desempenho está diretamente relacionado ao conceito de atributo de valor, posto que o objetivo de desempenho interfere diretamente nas características do produto, tais como a durabilidade, 0 tempo de entrega, o preço, entre outras. Todavia, verifica-se uma lacuna na literatura de estratégia de manufatura ao não reconhecer os aspectos de valor relacionados à subjetividade, estando voltada sobretudo para os aspectos concretos do produto. Isso provavelmente ocorre porquê estes aspectos estão relacionados principalmente às funções $M \& V$ e $P \& D$, que devem estar atentas a aspectos como o conteúdo valorativo do produto, a identificação com determinado grupo social, o conteúdo informacional, dentre outros. Contudo, sendo o valor do produto uma junção tanto de aspectos concretos quanto subjetivos, fica clara a necessidade de interação destas três funções no processo de concepção de valor (FLEURY; FLEURY, 2003). Como destacado anteriormente, Fleury e Fleury (2003) consideram que toda estratégia competitiva deve ser baseada em uma das seguintes categorias: excelência operacional, inovação em produto, ou relação com o cliente. A partir desta perspectiva é proposto o conceito de funções geradoras de valor, sendo elas, as funções: manufatura, $M \& V$ e $P \& D$. Esta denominação decorre do fato que estas três funções estão diretamente relacionadas à geração de valor dos produtos de uma firma.

\section{MÉTODO}

O objetivo deste artigo é analisar o papel das funções manufatura, P\&D e $M \& V$ no processo de geração de valor de manufaturas calçadistas. De forma mais 
específica, pretendeu-se identificar as relações existentes entre estas funções e o valor gerado pelo produto, considerando o desempenho de seus atributos de valor.

O objeto deste estudo consiste em três manufaturas de calçados masculinos (MCs), localizadas em Franca, interior do estado de São Paulo. Estas três empresas foram selecionadas em um estudo realizado em dezesseis MCs. Este recorte deu-se pelo fato destas empresas melhor representarem neste universo as diferenças existentes entre o composto de valor priorizado e a configuração das funções geradoras de valor. Elas serão denominadas G1, P1 e P2, sendo o termo inicial um denominador de porte, em que $G$ indica que a empresa é de grande e $P$ de pequeno porte. A Empresa $G$ foi considerada de grande porte por possuir dois mil e cem (2100) funcionários, enquanto as empresas P1 e P2 foram consideradas de pequeno porte, por possuírem respectivamente oitenta e seis (86) e quarenta (40) funcionários. O volume de produção é medido em pares de calçados fabricados diariamente. Estas empresas fabricam respectivamente em média doze mil (12000), seiscentos (600) e trezentos e cinquenta (350) pares. O uso de múltiplos casos como forma de caracterizar um determinado estudo dá-se mediante a possibilidade de análise comparativa (MEREDITH, 1998). Neste estudo as análises comparativas realizadas permitiram verificar como a ênfase da empresa em diferentes atributos de valor influencia a configuração das funções geradoras de valor. Estes casos também permitiram delinear um modelo conceitual das relações existentes entre a configuração de determinado aspecto de uma função geradora de valor e a performance de determinado atributo de valor do calçado fabricado pela MC.

Tendo em vista o objetivo da pesquisa o estudo de caso mostrou-se adequado por permitir identificar qual o papel das funções geradoras de valor na performance dos atributos de valor do calçado. O uso do estudo de caso para este fim está em consonância com textos como Miguel (2007) e Voss et al. (2002) que consideram que este método é adequado a análises qualitativas, permitindo identificar as relações entre os elementos da realidade que se pretende compreender.

As etapas para a realização da pesquisa foram: a elaboração do roteiro de pesquisa, o estabelecimento de contato com as MCs, as entrevistas, a transcrição e a análise dos dados. A elaboração do roteiro de pesquisa foi baseada na revisão de literatura, no contato prévio dos pesquisadores com a indústria calçadista, e no próprio contato inicial com as MCs em estudos piloto. Isso permitiu identificar quais 
os atributos de valor do calçado são mais relevantes nesta indústria (conforto; durabilidade; imagem - sintonia com a moda; e imagem - conceito de saúde) que serão apresentados nas próximas seções. Baseado em uma listagem adquirida de um sindicato patronal local, foi estabelecido contato com diversas MCs, em que aceitaram participar da pesquisa um total de dezesseis As três empresas apresentadas neste artigo são representativas dos três grupos estratégicos encontrados. A coleta de dados foi realizada por meio de entrevistas in loco nas MCs, com profissionais da alta administração, sendo gravadas e posteriormente transcritas. Na Empresa G foi entrevistado o diretor da função M\&V e nas empresas P1 e P2 os seus proprietários, que são os fundadores das mesmas e que exercem nelas o papel de comando. As entrevistas foram conduzidas com o auxílio de um roteiro de pesquisa com questões predominantemente abertas, segundo as recomendações de Yin (1994), em que procurou-se identificar os atributos de valor priorizados nos calçados destas empresas e a configuração de aspectos das suas funções geradoras de valor.

Considerando que o principal instrumento de coleta de dados foi a entrevista a profissionais da alta gerência das MCs pesquisadas, a confiabilidade dos dados baseia-se na sintonia das respostas destes profissionais com a realidade vivida por suas empresas. De modo a obter a maior objetividade nas respostas procurou-se identificar aspectos concretos, tanto na configuração dos atributos de valor priorizados pela empresa, quanto na forma com que ela configurava as suas funções geradoras de valor. Por exemplo, o uso de couro de alto padrão de qualidade (fator concreto associado à função manufatura) influencia o conforto do calçado, que é um atributo de valor. Já o uso de couro de classificação inferior, que é mais barato, denota o enfoque no baixo preço. Da perspectiva dos pesquisadores a gravação das entrevistas e a sua posterior transcrição permitiu conservar a totalidade das informações apresentadas pelos entrevistados.

O ponto central de toda a discussão do artigo é a relação entre a gênese de valor dos produtos das MCs e a configuração de aspectos relativos às funções geradoras de valor (manufatura, $M \& V$ e $P \& D$ ). Este modelo baseou-se nos construtos: atributos de valor, porte e funções geradoras de valor. A validade interna deste modelo restringe-se às três MCs pesquisadas em que foi possível identificar relações entre os atributos de valor priorizados por elas e a configuração das suas funções geradoras de valor. 
Destarte, o modelo de construtos apresentado transcende estas três empresas, conferindo validade externa à pesquisa, podendo ser aplicado futuramente a estudos de natureza quantitativa na indústria calçadista, uma vez que eles são comuns às empresas desta indústria.

\section{O PROCESSO DE GERAÇÃO DE VALOR NAS EMPRESAS PESQUISADAS}

Esta seção está subdividida nas subseções 4.1, 4.2 e 4.3 em que respectivamente são apresentados os dados coletados nas empresas G, P1 e P2 acerca dos atributos de valor priorizados por elas e a forma com que configuram suas funções geradoras de valor.

\subsection{A geração de valor na empresa G}

Conforme o Quadro 2 os atributos de valor priorizados pela Empresa G em seus calçados são: o conforto, a durabilidade, a imagem e a sintonia com a moda. Além destes atributos também foram considerados os meios que ela utiliza para reduzir seus custos, tendo em vista a sua importância na formação do preço do calçado e na lucratividade. A identificação dos atributos de valor priorizados por esta e pelas demais empresas pesquisadas foi feita segundo as atividades que sinalizassem isso nos relatos dos entrevistados. Com efeito, a priorização do conforto e da durabilidade do calçado baseia-se no uso de couro de alto padrão, no desenvolvimento próprio do calçado e no uso de solado customizado. A Empresa G possui uma função P\&D dedicada ao desenvolvimento de novos modelos de calçado, em que trabalham profissionais designers, que definem a forma do calçado (design) e modelistas, responsáveis pelo desenvolvimento do protótipo. Em relação ao solado, ela o desenvolve em parceria com um grande fabricante de solado local, essa medida ocorre pelo fato desta empresa possuir domínio de tecnologia no desenvolvimento de solado com características (uso de materiais, leveza e forma), que não poderiam ser reproduzidas internamente com a mesma performance.

Denotam a atenção da Empresa $G$ à imagem de marca a realização de campanhas publicitárias em que participam celebridades nacionais, a divulgação por meio de outdoors nas localidades em que seus calçados são vendidos, de revistas nacionais e também a participação de feiras de negócios nacionais e internacionais. 
O desenvolvimento próprio do calçado e o solado customizado também denotam a intenção da empresa em desenvolver uma identidade própria para seus produtos. Por fim, o seu porte lhe permite realizar maiores investimentos na divulgação da imagem do que empresas menores, uma vez que os mesmos podem ser diluídos no alto volume de calçados produzidos.

A procura de sintonia com a moda é favorecida pela compra de couro em estágio inicial de beneficiamento e o seu alto padrão de acabamento. Esta medida, além de possibilitar menor custo permite à Empresa $\mathrm{G}$ maior diversidade de cores $\mathrm{e}$ texturas de couro acabado. A participação dos profissionais de projeto da Empresa $\mathrm{G}$ de feiras de negócios, sobretudo as internacionais, permite que eles mantenham contato com as tendências vigentes na moda. O desenvolvimento próprio do calçado e o uso de solado customizado, inspirados na moda internacional, permitem a sintonia com a moda e a formação de identidade de marca. Novamente estas medidas são viabilizadas pelo porte desta empresa e são aparentemente inacessíveis às empresas menores.

Em relação ao custo de obtenção do produto final, a compra do couro em estado anterior ao acabado permite também à Empresa $\mathrm{G}$ a obtenção de economias, isso porque ela elimina com isso agentes intermediários nesta atividade, bem como aufere economias de escala, referentes ao volume negociado.

A descentralização produtiva da Empresa G ocorre de duas formas, ela possui uma unidade dedicada ao pesponto (costura da parte superior do calçado) em uma cidade situada próximo à Franca, a segunda está na manutenção de uma unidade dedicada a toda a produção de calçado localizada em um estado do nordeste brasileiro. Ela adota estas duas medidas para reduzir principalmente os custos de mão de obra. O uso de parte dos equipamentos com recursos computacionais e a presença de programas de melhoria da qualidade no chão de fábrica são medidas que visam aumentar a produtividade. Salvo o uso de programas de qualidade, estes vários meios utilizados pela Empresa G para reduzir os custos estão intimamente associados ao seu porte, sendo inacessíveis às empresas menores. 
Quadro 2 - Atributos de valor priorizados pela Empresa $G$ e fatores relacionados

\begin{tabular}{|c|c|c|c|}
\hline $\begin{array}{l}\text { Atributo de } \\
\text { Valor }\end{array}$ & Fator Relacionado & Função & Forma de influência \\
\hline \multirow{3}{*}{$\begin{array}{l}\text { Confortol } \\
\text { Durabilidad } \\
\text { e }\end{array}$} & Couro de alto padrão & Manufatura & Permite maior maciez e resistência \\
\hline & $\begin{array}{l}\text { Desenvolvimento próprio } \\
\text { do calçado }\end{array}$ & \multirow[t]{2}{*}{$P \& D$} & \multirow[t]{2}{*}{$\begin{array}{l}\text { Permite maior controle e teste } \\
\text { sobre o produto }\end{array}$} \\
\hline & Solado customizado & & \\
\hline \multirow{8}{*}{$\begin{array}{l}\text { Imagem - } \\
\text { Sintonia } \\
\text { com a } \\
\text { moda }\end{array}$} & $\begin{array}{l}\text { Compra do couro em } \\
\text { estágio inicial de } \\
\text { beneficiamento }\end{array}$ & \multirow[t]{2}{*}{ Manufatura } & $\begin{array}{l}\text { Permite a obtenção de maior } \\
\text { diversidade de cores e texturas de } \\
\text { couro }\end{array}$ \\
\hline & Couro de alto padrão & & $\begin{array}{l}\text { Permite maior variedade de cores e } \\
\text { texturas }\end{array}$ \\
\hline & $\begin{array}{l}\text { Campanhas publicitárias } \\
\text { com a participação de } \\
\text { celebridades }\end{array}$ & \multirow[t]{3}{*}{$M \& V$} & \multirow[t]{3}{*}{ Divulga a imagem } \\
\hline & $\begin{array}{l}\text { Publicidade visual } \\
\text { (outdoors; revistas) }\end{array}$ & & \\
\hline & $\begin{array}{l}\text { Participação em feiras } \\
\text { nacionais e internacionais }\end{array}$ & & \\
\hline & Porte & - & 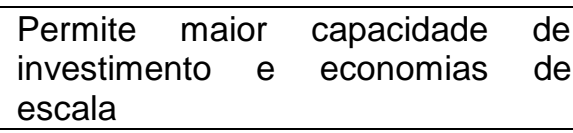 \\
\hline & $\begin{array}{l}\text { Desenvolvimento próprio } \\
\text { do calçado }\end{array}$ & \multirow[t]{2}{*}{$P \& D$} & \multirow[t]{2}{*}{ Criação de identidade de marca } \\
\hline & Solado customizado & & \\
\hline \multirow[t]{7}{*}{$\begin{array}{ll}\text { Custo } & \text { de } \\
\text { produção }\end{array}$} & $\begin{array}{l}\text { Compra do couro em } \\
\text { estágio inicial de } \\
\text { beneficiamento }\end{array}$ & \multirow[t]{4}{*}{ Manufatura } & $\begin{array}{l}\text { Elimina intermediários e permite } \\
\text { economias de escala na compra }\end{array}$ \\
\hline & $\begin{array}{l}\text { Descentralização } \\
\text { produtiva }\end{array}$ & & $\begin{array}{l}\text { Redução de custos com a mão de } \\
\text { obra }\end{array}$ \\
\hline & $\begin{array}{l}\text { Uso de equipamento } \\
\text { computadorizado }\end{array}$ & & \multirow[t]{2}{*}{ Permite aumentar a produtividade } \\
\hline & $\begin{array}{l}\text { Programa de gestão da } \\
\text { qualidade }\end{array}$ & & \\
\hline & $\begin{array}{l}\text { Desenvolvimento próprio } \\
\text { do calçado }\end{array}$ & \multirow{2}{*}{$P \& D$} & \multirow[t]{2}{*}{ Aumenta o custo final do calçado } \\
\hline & Solado customizado & & \\
\hline & \begin{tabular}{|l|} 
Porte \\
\end{tabular} & - & Permite obter economias de escala \\
\hline
\end{tabular}

Fonte: Os autores

De resto, embora a Empresa $G$ consiga reduzir custos conforme os itens acima, os investimentos relatados nas funções M\&V e P\&D aumentam os custos finais do calçado, comparando com uma situação em que eles não fossem realizados. Isso denota a intenção desta empresa em evitar a competição do seu calçado exclusivamente em preço, procurando nichos de mercado que deem importância a outros atributos de valor e que possivelmente the permita obter maior lucro. Comparando a proporção dos preços finais dos calçados das empresas G, P1 e P2, tem-se respectivamente os valores $0.72,1$ e 0.28 , o que dá embasamento a esta afirmação. 


\subsection{A geração de valor na empresa P1}

Verifica-se no Quadro 3 considerável semelhança entre a estratégia adotada pelas empresas P1 e G, pois ambas priorizam os mesmos atributos de valor, existindo também aspectos comuns nos fatores associados à performance dos mesmos. As principais diferenças encontram-se nos benefícios provenientes da maior escala, que somente a Empresa G pode auferir pelo seu maior porte.

Quadro 3 - Atributos de valor priorizados pela Empresa P1 e fatores relacionados

\begin{tabular}{|c|c|c|c|}
\hline $\begin{array}{l}\text { Atributo de } \\
\text { Valor }\end{array}$ & Fator Relacionado & Função & Forma de influência \\
\hline \multirow{3}{*}{$\begin{array}{l}\text { Conforto } \\
\text { e Durabilidade }\end{array}$} & Couro de alto padrão & Manufatura & Permite maior maciez e resistência \\
\hline & $\begin{array}{l}\text { Desenvolvimento próprio } \\
\text { do calçado }\end{array}$ & \multirow[t]{2}{*}{ P\&D } & \multirow[t]{2}{*}{$\begin{array}{l}\text { Permite formar maior controle e } \\
\text { teste sobre o produto }\end{array}$} \\
\hline & Solado customizado & & \\
\hline \multirow{5}{*}{$\begin{array}{l}\text { Imagem } \\
\text { Conceito de } \\
\text { saúde }\end{array}$} & Couro de alto padrão & Manufatura & Permite maior maciez e resistência \\
\hline & $\begin{array}{l}\text { Publicidade } \quad \text { visual } \\
\text { (outdoors) }\end{array}$ & \multirow[t]{2}{*}{$\mathrm{M} \& \mathrm{~V}$} & \multirow[t]{2}{*}{ Divulga a imagem } \\
\hline & $\begin{array}{l}\text { Participação em feiras } \\
\text { nacionais }\end{array}$ & & \\
\hline & $\begin{array}{l}\text { Desenvolvimento próprio } \\
\text { do calçado }\end{array}$ & \multirow[t]{2}{*}{$P \& D$} & \multirow[t]{2}{*}{ Criação de identidade de marca } \\
\hline & Solado customizado & & \\
\hline \multirow[t]{3}{*}{ Preço/Custo } & $\begin{array}{l}\text { Terceirização de } \\
\text { atividades }\end{array}$ & Manufatura & $\begin{array}{l}\text { Redução de custos com a mão de } \\
\text { obra }\end{array}$ \\
\hline & $\begin{array}{l}\text { Desenvolvimento próprio } \\
\text { do calçado }\end{array}$ & \multirow[t]{2}{*}{$P \& D$} & \multirow[t]{2}{*}{ Aumento do custo final do calçado } \\
\hline & Solado customizado & & \\
\hline
\end{tabular}

Fonte: Os autores

A ênfase da Empresa P1 no conforto e na durabilidade do calçado ocorre pelo uso de couro de alto padrão, desenvolvimento próprio e solado customizado. A priorização da imagem ocorre pela divulgação da marca por meio de outdoors nas cidades em que a empresa vende seus produtos, pela participação em feiras nacionais, pelo desenvolvimento próprio do calçado e pela aquisição de solado customizado. Ressalta-se que esta empresa procura associar o seu calçado ao conceito de saúde, por meio de atributos físicos (materiais leves, projeto próprio e solado customizado) e à leveza e alto conforto. Alguns dos seus calçados são direcionados à pessoas que necessitam utilizar calçados especiais, tanto por questões ortopédicas quanto pela necessidade de manter os pés intactos, por 
exemplo, os portadores de diabetes que devem evitar qualquer tipo de ferimento nos pés.

Como forma de reduzir custo a Empresa P1 terceiriza o pesponto do calçado, que é uma atividade intensiva em mão de obra, para agremiações locais conhecidas como bancas de pesponto. Todavia, esta empresa arca com maiores custos provenientes do desenvolvimento próprio do calçado e do solado customizado. Isso provavelmente contribui para que o seu calçado seja em média o mais caro, se comparado com os calçados das outras duas MCs apresentadas. Em relação ao solado, este é fabricado por um grande agente local (o mesmo que fabrica solado para a Empresa G.

\subsection{A geração de valor na empresa P2}

De acordo com o Quadro 4 o atributo de valor priorizado pela Empresa P2 é o baixo preço. Dentre os meios utilizados para reduzir o custo do produto e consequentemente seu preço, está o uso de couro de padrão inferior de qualidade, de materiais acessórios de baixo custo e o da terceirização do pesponto. Em relação ao desenvolvimento à Empresa P2 adquire o projeto de novos modelos de calçados de projetistas independentes (free lancers) e adquire solado padronizado, ou seja, um tipo de solado vendido no mercado local, e que outros fabricantes de calçado também podem ter acesso. Em relação à função $M \& V$ a empresa não realiza nenhum tipo de divulgação.

Quadro 4 - Atributos de valor priorizados pela Empresa P2 e fatores relacionados

\begin{tabular}{|l|l|l|l|}
\hline $\begin{array}{l}\text { Atributo de } \\
\text { Valor }\end{array}$ & Fator Relacionado & Função & Forma de influência \\
\hline Preço/custo & Couro de baixo padrão & Manufatura & $\begin{array}{l}\text { Redução do custo final do } \\
\text { produto }\end{array}$ \\
\cline { 2 - 2 } & $\begin{array}{l}\text { Compra de solado } \\
\text { padronizado }\end{array}$ & & Reduz o custo da mão de obra \\
\cline { 2 - 4 } & Terceirização & $\begin{array}{l}\text { Redução do custo final do } \\
\text { produto }\end{array}$ \\
\cline { 2 - 4 } & $\begin{array}{l}\text { Desenvolvimento externo do } \\
\text { calçado }\end{array}$ & P\&D & \\
\cline { 2 - 4 } & $\begin{array}{l}\text { Não foi relatado investimento } \\
\text { em divulgação }\end{array}$ & M\&V & \\
\hline
\end{tabular}

Fonte: Os autores. 


\section{ANÁLISE DOS RESULTADOS}

Diante dos dados das três MCs pesquisadas conclui-se que a performance de determinado atributo de valor é resultado da configuração das três funções geradoras de valor, bem como da política de aquisição de materiais. Com efeito, uma síntese das relações encontradas é apresentada no Quadro 4, derivada dos dados apresentados na seção anterior.

Foram identificados três tipos de relações denominadas da seguinte maneira: a letra $\mathrm{R}$ indica que a função possui influência direta no desempenho do atributo de valor; NR significa que não foi encontrada a relação direta; e RI, indica uma relação inversa. A relação inversa foi encontrada somente para o não investimento nas funções $M \& V$ e P\&D. Isso significa que fazer baixos investimentos nestas funções contribui para reduzir o preço do produto, que consiste na melhoria da performance deste atributo.

A priorização dos atributos de valor conforto e durabilidade do calçado está relacionada a fatores das funções manufatura e $P \& D$, sendo comuns às duas empresas que os priorizam (G e P1).

Quadro 5 - Síntese dos atributos de valor priorizados pelas MCs e funções relacionadas

\begin{tabular}{|c|c|c|c|c|c|}
\hline Empresa & $\begin{array}{l}\text { Atributo de } \\
\text { Valor }\end{array}$ & Manufatura & $M \& V$ & P\&D & Porte \\
\hline \multirow[t]{2}{*}{ G } & $\begin{array}{l}\text { Conforto } \\
\text { durabilidade }\end{array}$ & $\mathrm{R}$ & NR & $\mathrm{R}$ & - \\
\hline & \begin{tabular}{|lr} 
Imagem & - \\
Sintonia & com \\
a moda & \\
\end{tabular} & $\mathrm{R}$ & $\mathrm{R}$ & $\mathrm{R}$ & $\mathrm{R}$ \\
\hline \multirow[t]{2}{*}{ P1 } & $\begin{array}{l}\text { Conforto e } \\
\text { durabilidade }\end{array}$ & $\mathrm{R}$ & NR & $\mathrm{R}$ & \\
\hline & $\begin{array}{|ll|}\text { Imagem - } \\
\text { Conceito de } \\
\text { saúde }\end{array}$ & $\mathrm{R}$ & $\mathrm{R}$ & $\mathrm{R}$ & \\
\hline P2 & Preço baixo & $\mathrm{R}$ & $\mathrm{RI}$ & $\mathrm{RI}$ & \\
\hline
\end{tabular}

Fonte: Os autores

Embora as empresas G1 e P1 priorizem o conforto e a durabilidade elas possuem diferenças em aspectos relativos à apresentação da imagem de seus produtos. A Empresa $\mathrm{G}$ procura transmitir a imagem de calçado em sintonia com a 
moda. Neste caso além do projeto próprio e do uso do solado customizado, há uma participação ativa desta empresa de feiras internacionais, como já relatado. Neste caso, o porte desta empresa favorece este comportamento, uma vez que ele exige alto investimento. Provavelmente seja este o motivo pelo qual a Empresa P1 embora invista na divulgação de sua imagem, a direcione a um segmento específico de clientes, aquele que possuí necessidades especiais no uso do calçado e/ou que valorize a sua associação com o conceito de saúde. Verificou-se que esta empresa prioriza mais o conforto do calçado do que seu padrão estético (sintonia com a moda), o que deve-se principalmente a aspectos relativos ao projeto próprio do calçado e ao fato dela não manter contato direto com as tendências da moda internacional.

A priorização dos atributos durabilidade, conforto e imagem do produto pelas empresas G e P1 sugere a necessidade de uma ação sistêmica das três funções geradoras de valor. O maior desenvolvimento organizacional destas empresas no que tange a constituição das funções $M \& V$ e $P \& D$ lhes permite priorizar atributos diferentes do preço e evitar a competição centrada neste atributo de valor. Considerando-se que as estas empresas poderiam fabricar calçados de baixo preço, utilizando as mesmas práticas da Empresa P2, com destaque para a empresa G capaz de obter ganhos de escala em vários aspectos, elas provavelmente focam outros atributos de valor, por ser esta uma estratégia mais lucrativa e estável ao buscar a fidelidade do cliente.

O papel ativo das funções P\&D e M\&V na geração de valor dos calçados das empresas G e P1 coaduna-se com os achados de Garcia et al. (2004) que na época de sua pesquisa ao investigarem as relações de governança entre agentes da indústria calçadista de Franca e agentes internacionais, constataram que estes últimos mantinham o controle das atividades de $P \& D$ e $M \& V$, enquanto transferiam para os agentes locais as atividades puramente produtivas.

Já a oferta de calçados pela Empresa P2 a preços baixos ocorre mediante uma estrutura de gênese de valor centrada apenas na função manufatura, portanto não fazendo investimentos nas funções $M \& V$ e P\&D. Deve-se citar também neste caso o uso de couro e de materiais de baixo padrão de qualidade, de solado padronizado e da terceirização. Estas práticas contrapõem o tradicional pressuposto de textos de estratégia e organização industrial (ex. KUPFER; HASENCLEVER, 
2002; PORTER, 1986) em que o principal mecanismo para a redução de custos consiste nas economias de escala.

Observou-se que independentemente dos atributos de valor priorizados, todas as MCs implementam medidas para a redução de custos, uma vez que o custo está associado diretamente à lucratividade do negócio.

A Empresa $G$ possui o maior leque de possibilidades estratégicas provenientes da sua capacidade de auferir economias de escala e implementar políticas de valorização da imagem e da moda, inacessíveis às MCs de menor porte. Por outro lado, um horizonte mais estreito de possibilidades estratégicas destas empresas não necessariamente torna determinística a sua estratégia. No caso da Empresa P1, uma alternativa encontrada foi priorizar a oferta de calçados de alto valor agregado, todavia, com um composto de valor ligeiramente diferente daquele oferecido pela Empresa $\mathrm{G}$, de modo a justificar o maior preço cobrado. Esta situação denota que além do porte, aspectos como o conhecimento e as competências acumuladas ao longo do tempo no negócio são importantes na definição das estratégias das firmas.

A conclusão acima coaduna-se com Cuervo-Cazurra e Un (2010) que verificaram que o não investimento de empresas em $P \& D$ além de estar correlacionado com a baixa disponibilidade financeira, também está associado ao conhecimento adquirido pelas mesmas. No presente estudo a menor capacidade financeira das empresas P1 e P2 não implicou nas mesmas escolhas estratégicas. A estratégia de produzir calçados de alto valor agregado da Empresa P1 também foi baseada no conhecimento de seu fundador, que antes de estabelecer esta firma acumulou experiência na exportação e importação de calçado, sendo inclusive grande conhecedor do mercado europeu.

Foi também observado que os fatores de custos das MCs são de várias naturezas, tais como, os investimentos nas funções geradoras de valor, a escala, a eficiência do processo produtivo, a qualidade das matérias-primas utilizadas e que as desvantagens de custo enfrentadas por uma empresa em determinados aspectos, podem ser compensadas na configuração de outros. A evidência disto está na Empresa P2, que possuindo desvantagens de escala, procurou a redução de custos por outros meios já descritos anteriormente. Por fim, a configuração das funções geradoras de valor é algo realizado sob o escopo da estratégia competitiva da empresa. Todavia, a classificação das estratégias em diferenciação, baixo custo 
e enfoque proposta por Porter (1986) não se mostra suficiente para a compreensão detalhada deste processo nas empresas atuais; embora as estratégias das empresas G e P1 possam ser classificadas como diferenciação, existem consideráveis diferenças entre elas, denotando a necessidade de uma visão mais detalhada de suas estratégias, para se entender melhor o seu cenário competitivo. Existem indícios que diversos atributos de valor de um produto são resultantes da ação das três funções (manufatura, M\&V, P\&D), todavia, a participação de cada uma no desempenho de cada atributo normalmente variará.

O conceito de funções geradoras de valor permite propor um modelo de relacionamento entre as estratégias no contexto da firma, expresso na Figura 1. No nível funcional estão as estratégias das funções geradoras de valor que devem dar suporte à estratégia competitiva da UEN (unidade estratégica de negócio). Frente ao exposto, a forma com que a UEN, ou a firma individual irá competir, ou seja, o valor gerado, está diretamente associado à configuração e a forma de funcionamento destas funções. Cabe às funções de apoio (recursos humanos, finanças e contabilidade,.....) darem suporte às funções geradoras de valor. Esta nova forma de conceber as estratégias no contexto da UEN, alinha-se com Chakravarthy e Henderson (2007) que defendem a necessidade de novas formas de hierarquização das estratégias, para que as firmas possam melhor enfrentar os cenários competitivos atuais. 
Figura 1 - Relações entre as estratégias funcionais e competitiva

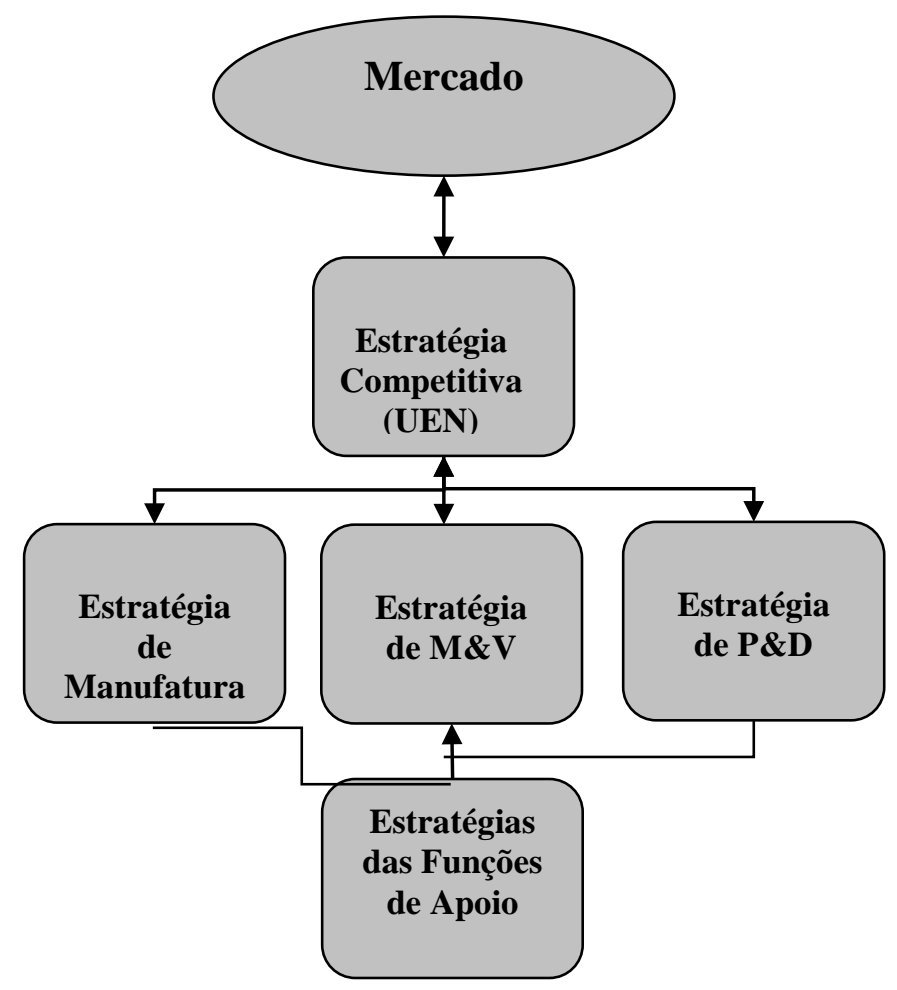

Fonte: Os autores.

\section{CONCLUSÃO}

Este artigo discute o papel das funções manufatura, M\&V e P\&D na geração de valor dos produtos de uma empresa. Questão que foi validada empiricamente pelos três estudos de casos em manufaturas calçadistas. Embora este estudo não permita generalizações estatísticas, foi possível identificar relações no tocante a configuração das funções geradoras de valor e a performance de determinado atributo de valor do calçado.

Ressalta-se que, salvo o preço, todos os demais atributos de valor tiveram sua performance decorrente da ação de duas ou mais funções geradoras de valor. Com efeito, a elaboração da estratégia destas três funções em conjunto e não de estratégias funcionais isoladas pode permitir a obtenção de maior sinergia e de benefícios diretos para o processo de geração de valor nos produtos de uma firma.

Embora o maior porte implique no maior leque de possibilidades estratégicas, a MC de pequeno porte não está confinada à estratégia de baixo preço. $O$ fato da 
Empresa P1 explorar mercados de alto valor agregado evidencia que além do porte, outros recursos, como conhecimento e experiências acumuladas são determinantes nas possibilidades estratégicas da empresa.

Em síntese, este artigo apresenta as seguintes contribuições: constata nas empresas estudadas que a estratégia de baixo custo baseia-se em aspectos da função manufatura e no baixo investimento nas funções P\&D e M\&V e a estratégia de calçados de alto valor agregado exige ênfase conjunta nas três funções geradoras de valor; apresenta uma visão objetiva das relações entre a configuração de aspectos das funções geradoras de valor e a performance dos atributos de valor do calçado; apresenta por meio do conceito de atributo de valor, uma proposta geral para a compreensão da estratégia competitiva da empresa, ao mesmo tempo que permite a aplicação deste conceito na análise de qualquer situação específica; evidencia pelo estudo empírico o pressuposto que a estratégia competitiva molda a estrutura empresarial, corroborando textos como Chandler (1990) e Pinto et al. (2010).

Como possibilidade de pesquisas futuras pode-se: desenvolver uma metodologia de análise de valor e elaboração da estratégia das funções geradoras da firma; buscar uma melhor compreensão das formas de geração de valor de empresas em vários ramos de atividade; aplicar os conceitos desenvolvidos neste artigo em uma pesquisa de cunho estatístico na indústria calçadista.

\section{REFERÊNCIAS}

ABE, C. F. J.; CARVALHO, M. M. Alinhamento entre estratégia e critérios competitivos: um estudo em empresa do setor de bebidas. Revista Produção Online, v.11, n.1, p.135-161, 2011. dx.doi.org/10.14488/1676-1901.v11i1.537

ADNER, R.; ZEMSKY, P. A demand-based perspective on sustainable competitive advantage. Strategic Management Journal, v.27, n.3, p.215-239, 2006.

dx.doi.org/10.1002/smj.513

ALVES FILHO, A. G.; NOGUEIRA, E.; BENTO, P.E.G.; Análise das estratégias de produção de seis montadoras de motores para automóveis. Gestão \& Produção, v.18, n.3, p.603-

618, 2011. dx.doi.org/10.1590/S0104-530X2011000300012.

ANSOFF, H. I. A nova estratégia empresarial. São Paulo: Atlas, 1990. 
AUGER, P.; D. EVINNEY, T. M.; LOUVIERE, J. J.; BURKE, P. F. The importance of social product attributes in consumer purchasing decisions: a multi-country comparative study. International Business Review, v.19, n.2, p.140-159, 2010.

dx.doi.org/10.1016/j.ibusrev.2009.10.002

BABIN, B. J. DARDEN, W. R.; GRIFFIN, M. Work and/or fun: measuring hedonic and utilitarian shopping value. Journal of Consumer Research, v.20, n.4, p.644-656, 1994 dx.doi.org/10.1086/209376

BACHEGA, S. J.; GODINHO FILHO, M. Identificação de foco estratégico e de consistência entre fins e meios em empresas calçadistas do Estado de São Paulo. Gestão \& Produção, v.18, n.2, p.391-408, 2011. dx.doi.org/10.1590/S0104-530X2011000200013

BARNEY, J. B. Firm resources and sustained competitive advantage. Journal of Management, v.17, n.1, p.99-120, 1991. dx.doi.org/10.1177/014920639101700108

BORCHARDT, M.; WENDT, M.H.; SELLITTO, M.A.; PEREIRA, G. M. Reprojeto do contraforte: um caso de aplicação do ecodesign em manufatura calçadista. Produção, v.20, n.3, p.392-403, 2010.dx.doi.org/10.1590/S0103-65132010005000006

BORCHARDT, M.; WENDT, M.H.; SELLITTO, M.A.; PEREIRA, G. M. Avaliação da presença de práticas do design for environment (DFE) no desenvolvimento de produto de uma empresa da indústria química. Produção, v.22, n.1, p.58-69, 2012.

dx.doi.org/10.1590/S0103-65132012005000001

BROWMAN, C.; AMBROSINI,V. Firm value creation and levels of strategy. Management Decision,v.45, n.3, p.360-371, 2007.dx.doi.org/10.1108/00251740710745007

BROWMN, S.; BLACKMON, K. Aligning manufacturing strategy and business-level competitive strategy in new competitive environments: the case of strategic resonance.

Journal of Management Studies, v.42, n.4, p.793-815, 2005.

dx.doi.org/10.1111/j.1467-6486.2005.00519.x

CALIFE, N. F. S.; NOGUEIRA, E.; ALVES FILHO, A. G.Empresas do setor de linha branca e suas estratégias competitivas e de produção. Revista Produção Online, v.10, n.2, 2010. dx.doi.org/10.14488/1676-1901.v10i2.293

CACHON, G.; SWINNEY, R. The value of fast fashion: quick response, enhancing design, and strategic consumer behavior. Management Science, v.57, p.778-795, 2011.

dx.doi.org/10.1287/mnsc.1100.1303

CHAKRAVARTHY, B.; HENDERSON, J. From a hierarchy to a heterarchy of strategies: adapting to a changing context. Management Decision, v.45, n.3, p.642-652, 2007. dx.doi.org/10.1108/00251740710745160

CHANDLER, A. Strategy and Structure: chapters in the history of the industrial enterprise. Cambridge, MA: MIT Press, 1990.

CONTADOR, J. C. Modelo para aumentar a competitividade industrial. Edgard Blucher, 2003.

CRONIN, J. Jr.; BRADY, M.; BRAND, R. R.; HIGHTOWER, R. Jr.; SHEMWELL, D. A crosssectional test of the effect and conceptualization of service value. Journal of Services Marketing, v.11, n.6, p.375-391, 1997. dx.doi.org/10.1108/08876049710187482 
CUERVO-CAZURRA, A.; UN, A.C. Why some firms never invest in formal R\&D. Strategic Management Journal, v.31, p.759-779, 2010. dx.doi.org 10.1002/smj.836

FINNEY, R. Z.; LUEG, J. E.; CAMPBELL, N. D. Market pioneers, late movers, and the resource based view (RBV): a conceptual model. Journal of Business Research, v.61, n.9, September, p.925-932, 2008. dx.doi.org/10.1016/j.jbusres.2007.09.023

FLEURY, A. C. C.; FLEURY, M. T. L. Estratégias Competitivas Essenciais: Perspectivas Para a Internacionalização da Indústria no Brasil. Gestão \& Produção, v.10, n.2, p.129-144, 2003. dx.doi.org/10.1590/S0104-530X2003000200002.

GARCIA, R.; MOTTA, F. G.; AMATO NETO, J. Uma análise das características da estrutura de governança em sistemas locais de produção e suas relações com a cadeia global.

Gestão \& Produção, v.11, n.3, p.343-354, 2004. dx.doi.org/10.1590/S0104-

530X2004000300008.

GODINHO FILHO, M.; FERNANDES, F. C. F. Paradigmas Estratégicos de Gestão da Manufatura (PEGEMs): elementos-chave e modelo conceitual. Gestão \& Produção, v.12, n.3, p.333-345, 2005. dx.doi.org/10.1590/S0104-530X2005000300005.

GUIMARÃES, M. R. N.; TEODORO FILHO, A. M.; LARA, F. F.; SALTORATO, P. Estratégia de produção na indústria de autopeças: estudo de multicasos em empresas da região de sorocaba. Revista Produção Online, v.14, n.2, p.499-532, 2014.

dx.doi.org/10.14488/1676-1901.v14i2.1364

HALLGREN, M.; OLHAGER, J. Differentiating manufacturing focus. International Journal of Production Research, v. 44, n. 18-19, p. 3863-3878, 2006.

dx.doi.org/10.1080/00207540600702290

HAYES, R. H.; WHEELWRIGHT, S. C. Restoring our competitive edge: competing through manufacturing. New York: John Wiley, 1984.

HILL, T. Manufactoring strategy: text and cases. $2^{\text {nd }}$ ed., Burr Ridge: Irwin, 1994.

JOSEPH, J.; OCASIO, W. Architecture, attention, and adaptation in the multibusiness firm: General electric from 1951 to 2001. Strategic Management Journal. v.33, n.6, p.633-660. 2012. dx.doi.org/10.1002/smj.1971

KALRA, A.; SHI, M.; SRINIVASAN, K. C. Salesforce competition scheme and consumer inferences. Management Science, v.49, p.655-672, 2003.

dx.doi/abs/10.1287/mnsc.49.5.655.15146

KIM, K.; CHHAJED, D. Product Design with Multiple Quality-Type Attributes. Management Science, v.48, p.1502-1511, 2002. dx.doi.org/10.1287/mnsc.48.11.1502.265

KLIPPEL, M. A. J.; VALLE, J. A.; LAUREANO, E. Estratégia de produção em empresas com linhas de produtos diferenciadas: um estudo de caso em uma empresa rodoferroviária.

Gestão \& Produção, v.12, n.3, p.417-428, 2005.

dx.doi.org/10.1590/S0104-530X2005000300011

KUPFER, D.; HASENCLEVER, L. Economia industrial: fundamentos teóricos e práticas no Brasil. Rio de Janeiro-Campus. 2002. 
MaCARTHY, B.L.; FERNANDES, F.C.F. A multi-dimensional classification of production systems for the design and selectin of production planning and control systems. Production Planning \& Control, v. 11, n. 5, p. 481-496, 2000. dx.doi.org/10.1080/09537280050051988

MAIA, J. L.; CERRA, A. L.; ALVES FILHO, A. G. Inter-relações entre estratégia de operações e gestão da cadeia de suprimentos: estudos de caso no segmento de motores para automóveis. Gestão \& Produção, v.12, n.3, p.377-391, 2005.

dx.doi.org/10.1590/S0104-530X2005000300008.

MEREDITH, J. Building operations management theory through case and field research. Journal of Operations Management, v.16, p.441-454, 1998.

dx.doi.org/10.1016/S0272-6963(98)00023-0

MEREDITH, J. R.; McCUTCHEON, D. M.; HARTLEY, J. Enhancing competitiveness through the new market value equation. International Journal of Production and Operations Management, v.14, n.11, p.7-22, 1994.

dx.doi.org/10.1108/01443579410068611

MIGUEL, P. A. C. Estudo de caso na engenharia de produção: estruturação e recomendações para sua condução. Produção, v.17, n.1, p.216-229, 2007. dx.doi.org/10.1590/S0103-65132007000100015

OLAVARRIETA, S.; FRIEDMANN, R. Market orientation, knowledge-related resources and firm performance. Journal of Business Research, v.61, n.6, p.623-630, 2008. dx.doi.org/10.1016/j.jbusres.2007.06.037

PAIVA, E.; CARVALHO, L; FENSTERSEIFER, J. Estratégia de produção e de operações. Porto Alegre: Bookman, 2004.

PEHRSSON, A. Business relatedness and performance: a study of managerial perceptions. Strategic Management Journal, v.27, n.3, p.265-282, 2006.

dx.doi.org/10.1002/smj.516

PEIXOTO, M. G. M.; PINHEIRO, R. S.; NOGUEIRA,E.; BATALHA, M. O. Estratégias de Produção de Empresas de um Arranjo Produtivo Local: Aplicação do Modelo de Áreas de Decisão. Revista Produção Online, v.13, n.4, p.1517-1542, 2013.

dx.doi.org/10.14488/1676-1901.v13i4.1496

PEREIRA, G. M.; SELLITO, M. A.; BORCHARDT, M. Alterações nos fatores de competição da indústria calçadista exportadora devido à entrada de competidores asiáticos Produção, v. 20, n.2, p.149-159, 2010. dx.doi.org/10.1590/S0103-65132010005000022.

PERTUSA-ORTEGA, E. M.; MOLINA-AZORIN, J. F.; CORTÉS, E. C. Competitive strategies and firm performance: a comparative analysis of pure, hybrid and stuck in the middle strategies in spanish firms. British Management Journal, v.20, p.508-523, 2009. dx.doi.org/10.1111/j.1467-8551.2008.00597.x

PILKINGTON, A.; MEREDITH, J. The evolution of the intellectual structure of operations management - 1980 - 2006: A citation/co-citation analysis. Journal of Operations Management, v.27, p.185-202, 2009. dx.doi.org/10.1016/j.jom.2008.08.001 
PINTO, R. A. Q.; RAMOS, M. C.; CORSO, J. M. D. Relação entre a estrutura, a estratégia e a arquitetura estratégica como fator de sucesso: um estudo de caso. Revista Produção

Online, v.10, n.1, p. 2-25, 2010. $\underline{\mathrm{dx} . \text { doi.org/10.14488/1676-1901.v10i1.207 }}$

PIRES, S. R. I; AGOSTINHO, O. L. Estratégias competitivas e prioridades competitivas da manufatura: um estudo exploratório. Produção, v.4, n.1, p.23-32, 1994.

dx.doi.org/10.1590/S0103-65131994000100002.

PORTER, M. E. Estratégia competitiva. Rio de Janeiro: Campus, 1986.

PORTER, M. E. Vantagem competitiva: criando e sustentando um desempenho superior. Rio de Janeiro: Campus, 1989.

RONDA-PUPO, G. A.; GUERRAS-MARTIN, L. A. Dynamics of the evolution of the strategy concept 1962-2008: a co-word analysis. Strategic Management Journal, v.33, p.162-188, 2012. dx.doi.org/10.1002/smj.948

ROTH, A. V.; MILLER, J. G. A taxonomy of manufacturing strategies. Management Science, v.40, n.3, p.285-304, 1994. dx.doi.org/10.1287/mnsc.40.3.285

SANTALA, M.; PARVINEN, P. From strategic fit to customer fit. Management Decision, v.45, n.3, p.582-601, v.45, p.582-601, 2007. dx.doi.org//10.1108/00251740710745133

SANTOS, D. T.; BATALHA, M. O. Estratégia de produção em arranjos produtivos cerâmicos: o caso de Pedreira (SP). Revista Produção Online, v.10, n.3, 2010.

dx.doi.org/10.14488/1676-1901.v10i3.342

SELLITTO, M. A.; WALTER, C. Medição e pré-controle de desempenho de um plano de ações estratégicas. Gestão \& Produção, v.12, n.3, p.443-458,2005.

dx.doi.org/10.1590/S0104-530X2005000300013

SELLITTO, M. A.; WALTER, C. Avaliação do desempenho de uma manufatura de equipamentos eletrônicos segundo critérios de competição. Produção, v.16, n.1, p.34-47, 2006. dx.doi.org/10.1590/S0103-65132006000100004

SELLITTO, M. A.; BORCHARDT, M.; PEREIRA, G. M.; SILVA, M. Prioridades estratégicas em serviços de pós-venda de uma empresa de manufatura de base tecnológica. Gestão \& Produção, v.18, n.1, p.131-144, 2011. dx.doi.org/10.1590/S0104-530X2011000100010.

SILVA, E. M.; SANTOS, F. C. A. Análise do alinhamento da estratégia de produção com a estratégia competitiva na indústria moveleira. Produção, v.15, n.2, p.286-299, 2005.

dx.doi.org/10.1590/S0103-65132005000200012.

SILVA, B.; FINARDI, C.; FORNECK, M.; SELLITTO, M. A. Análise comparativa e avaliação de prioridades de competição em três cadeias de suprimentos do setor petroquímico.

Produção, v.22, n.2, p.225-236, 2012. dx.doi.org/10.1590/S0103-65132012005000015

SILVA, E. M.; SANTOS, F, C, A.Revisitando a estratégia de produção: as contribuições para um novo construto. Revista Produção Online, v.8,n.1, 2008.

dx.doi.org/10.14488/1676-1901.v8i1.27

SILVA, E. M.; SANTOS, F. C. A.; CASTRO, M. Os efeitos diretos e indiretos das práticas de produção sobre o desempenho: survey em firmas moveleiras no Brasil. RAE eletrônica, v.9, n.2, Art.9, 2010.dx.doi.org/10.1590/S1676-56482010000200005 
SILVA, E. M.; SANTOS, F. C. A.; CASTRO, M. Análise das relações entre estratégia de produção, práticas e desempenho operacional. Produção, v.22, n.0, p.0-0, (Ahead of print), 2012. dx.doi.org/10.1590/S0103-65132012005000009.

SKINNER, W. Manufacturing - missing link in corporate strategy. Harvard Business Review, p.136-144, may-june, 1969.

SKINNER, W.: The Focused factory. Harvard Business Review, v.52, n.3, p.113-121, MayJune, 1974.

SLACK, N. Vantagem competitiva em manufatura: atingindo a competitividade nas operações industriais. São Paulo: Atlas, 2002.

VARANDAS, JR.; A.; MIGUEL, P. A. C. Análise do processo de preparação da produção no desenvolvimento de novos produtos por meio de um estudo de caso em uma empresa do setor siderúrgico.Produção,v.22,n.1,p.185-200,2012.

dx.doi.org/10.1590/S0103-65132012005000012.

VOSS, C.; TSIKRIKTSIS, N.; FROHLICH, M. Case research in operations management. International. Journal of Operations and Productions Management, v.22, n.2, p.195-219, 2002. dx.doi.org/10.1108/01443570210414329

WHEELWRIGHT, S. C.: Manufacturing Strategy: Defining the Missing Link. Strategic Management Journal, v. 5, p. 77-91, 1984. dx.doi/10.1002/smj.4250050106

YIN, R. K. Case Study research: design and methods. Second Edition, SAGE Publications, 1994.

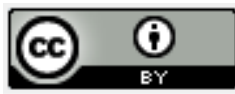

Artigo recebido em 26/02/2014 e aceito para publicação em 19/12/2014

DOI: http://dx.doi.org/ 10.14488/1676-1901.v15i1.1739 